\title{
Small scale simulations of wind-velocity and heat-fluxes of atmospheric geophysical flows with wind tunnel experiments: the STROMA collaboration
}

\author{
Bardo E. J. Bodmann ${ }^{1}$, Gervásio A. Degrazia², Otávio C. Acevedo², Acir M. Loredo-Souza', Marco T. M. B. de Vilhena ${ }^{1}$, \\ Silvia B. A. Rolim ${ }^{1}$, Marcelo R. de Moraes ${ }^{3}$, Giuliano Demarco², Leonardo B. Trindade ${ }^{4}$, Adrián R. Wittwer ${ }^{5}$, Rodrigo M. \\ Dorado ${ }^{1}$, Michael Beer ${ }^{6}$. \\ ${ }^{1}$ UFRGS, RS; ${ }^{2}$ UFSM, RS; ${ }^{3}$; UNIPAMPA, Bagé, RS, Brazil; ${ }^{4}$ STE Pesquisa e Desenvolvimento, RS; ${ }^{5}$ Universidad \\ Nacional del Nordeste, Argentina; ${ }^{6}$ Institute for Risk and Uncertainty-University of Liverpool, England.
}

\section{Copyright 2014, SBGf - Sociedade Brasileira de Geofísica}

Este texto foi preparado para a apresentação no VI Simpósio Brasileiro de Geofísica, Porto Alegre, 14 a 16 de outubro de 2014. Seu conteúdo foi revisado pelo Comitê Técnico do VI SimBGf, mas não necessariamente representa a opinião da SBGf ou de seus associados. É proibida a reprodução total ou parcial deste material para propósitos comerciais sem prévia autorização da SBGf.

\section{Abstract}

The study of geophysical flows in the planetary boundary layer is of fundamental importance to investigate the wind effect acting on man made constructions and the generation of wind energy. To this end the STROMA collaboration was organized with its first project that is designed to simulate wind velocity fields and heat fluxes in a model scale by wind tunnel experiments using the Laboratory of Construction Aerodynamics (LAC) at the Federal University of Rio Grande do Sul. The aim of this work is to present and discuss the STROMA project.

\section{Introduction}

The planetary boundary layer is probably the space in the atmosphere where interference between man made constructions and natural habitats is most striking. Not only populated areas but also agricultural and forest areas suffered from modifications that are significant or comparable to the scale of the planetary boundary layer height $\approx 10^{3} \mathrm{~m}$. For instance building heights of the order of $10^{2} \mathrm{~m}$ are common in large cities and latest generation wind turbines also surpass this extent. However, both construction categories influence the wind flow characteristics in different ways the firsts one predominantly passive, whereas the second type has an active contribution to this flow by virtue of moving blades.

Numerous efforts have been made for decades to get a quantitative understanding of the interplay of the dynamics of man made structures with those of natural habitats. Evidently a huge number of issues could be addressed in this context, nevertheless in this work we put focus on the question of simulating mechanical as well as thermal characteristics of flows that may mimic micro-meteorological scenarios and analyse the impact of passive and active obstacles on these flows. One evident aspect is the diverse turbulent regimes with its specific spectral characteristics and entropy production by either passive or moving obstacles. Moreover, we shall approach the crucial question of rescaling findings of wind tunnel experiments to real scale phenomena, which is a challenge since the simulated and evaluated phenomena are intrinsically highly non-linear.

To this end the STROMA collaboration was founded with its first project, that is designed to simulate wind velocity fields and heat fluxes in a model scale by wind tunnel experiments using the Laboratory of Construction Aerodynamics (LAC) at the Federal University of Rio Grande do Sul, (Porto Alegre, Rio Grande do Sul, Brazil). The LAC Wind Tunnel is a closed return low speed wind tunnel, specifically projected for dynamics and statics studies on civil construction models. Its design allows the simulation of the natural winds main characteristics. It has a length/height ratio in the main test section larger than 10 and dimensions of $1.30 \mathrm{~m} \times 0.90 \mathrm{~m} \times 9.32 \mathrm{~m}$ (width $\mathrm{x}$ height $x$ length). In this chamber the maximum of a large range of controllable wind speeds is $42 \mathrm{~m} / \mathrm{s}=150 \mathrm{Km} / \mathrm{h}$, which is for uniform flow conditions. It is noteworthy, that former activities of LAC contributed to the Brazilian Norm for wind-loads in building construction and structural design NBR-6123. The novelty of this experiment in comparison to the previous ones is, that the velocity fields may not only be conditioned to simulate a variety of turbulence regimes, i.e. mechanical aspects of the flow, but also allows for superimposing thermal effects, such as vertical heat flow from the lower surface of the chamber. To this end the LAC-UFRGS wind tunnel will be equipped with a heating or cooling system at the bottom part of the tunnel. The heating and cooling system will be monitored by measuring the surface temperature as well as the effective heat flux to or from the surface. The resulting thermal and mechanical system will then be used to create data for horizontal and vertical windprofiles equivalent to a height approximately $200 \mathrm{~m}$. Influences from the thermal stratification on the boundary layer will be analysed, effects of the orography, of roughness and of texture among others on the wind profile will be measured systematically. Furthermore, comparisons of the horizontal and vertical wind speed distributions with and without obstacles such as civil engineering constructions and wind-powergenerators are worked out. As a final issue the experimental findings shall indicate possible feedback effects on the simulated "micro-meteorology" in the wind tunnel. These results shall open pathways to predict properties of the wind-velocity field under specific mechanical and thermal conditions and moreover shall indicate consequences of the presence of man built structures on the flow. 


\section{Experiments}

Several scenarios will be studied such as the stratified flow over surfaces with uniform temperatures or with horizontal temperature gradients, with different roughness and texture compositions, with and without the presence of wind turbines. In this type of flow, the air is heated or cooled in layers, forming near the surface a stratified boundary layer, which implies in different regimes of stability, which are convective or neutral and in cases with horizontal temperature gradients generate spatial transients of turbulence. These types of layer shall replicate the first layer of the atmosphere, both during heating, that occurs during the day due to incident solar radiation on the earth's surface and cooling caused by the emission of infra-red radiation during the night.

So far wind tunnel studies examined turbulent flow under conditions of stable or neutral thermal stratification (Lienhard and Atta, 1990; Yoon and Warhaft, 1990; Ohya et al. 1997; Ohya, 2001; Ohya et al., 2008), but turbulence due to thermal effects that make use of controlled heat fluxes were not considered. The present proposal contemplates studies of horizontal and vertical profiles of turbulence measuring statistical moments, with emphasis to the question as how they are affected by thermal stratification. As a real scale counterpart, known similarity relations established for the the atmospheric boundary layer and obtained in field experiments are compared with laboratory results. Although there are experimental comparisons with theoretical predictions for flow in the planetary boundary layer, nothing is known about the influence of wind turbines on micro-meteorology. Hence, the series of experiments address distinct settings for thermal heating of the surface, surface cooling and horizontal temperature gradients together with the influence of different roughness of surfaces and finally with the presence of wind turbines and the fundamental question of how to relate them to findings of real scale field experiments.

The aerodynamic wind tunnel LAC-UFRGS is a closed system with air recirculation, variable turbulent intensity, with a circular experimental section of $800 \mathrm{~mm}$ diameter. A scheme is designed that will be hosted in this available space, so that the anemometry support system can be moved and positioned at different points in both vertical positions and in the direction of the flow. To obtain the effect of vertical thermal stratification an aluminium plate is installed at the floor of the experimental section with several Peltier elements attached. This set-up enables heating or cooling of the metal surface and also allows to define horizontal temperature gradients. To control the temperature of this plate a digital multimeter FLUKE SERIES 170 (179 TRUE RMS MULTIMETER) is used. The upstream wind speed (reference speed), which may be adjusted between $1 \mathrm{~m} / \mathrm{s}-10 \mathrm{~m} / \mathrm{s}$, is monitored with Pitot tubes connected to a barometer GE Sensing DPI 610 module. In addition to the wind speed a selection of

certified wind conditioners allows to establish a variety of boundary layer regimes that may be simulated, as for instance present in flat planes, pasture areas and sea sides among many others.
For data acquisition two different techniques are used: hot-wire anemometry together with temperature measurements and visualization by tracer substances. The first one will be used for the simultaneous collection of mean values as well as fluctuations of velocity and temperature fields. The optical capture of tracer movement is used to obtain a semi-quantitative description of the turbulent wind field profile.

A hot-wire anemometer consists of a heated filament which is exposed to the wind flow. This filament is connected to an electronic circuit that is able to monitor its electrical resistance variation resulting from the interaction with the flow. Using calibration data, one can establish a relation between flow velocity and resistance observed in the heated filament (Duda et al., 2006). For this study hot-wire sensors Dantec 55P15 and a sensor Dantec 56C01 are used to obtain simultaneously the velocity and temperature profiles, which is essential for data evaluation by virtue of the thermal contributions to the flow. Therefore a calibration of the sensor for velocity measurements with temperature compensation shall be applied. In order to interpret the data consistently the Dantec Streamline 90 N10 system consisting of three modules of hot-wire anemometer at constant temperature (90C10), and a calibration module (90H01) are employed Bruun (1996). The visualization technique for recording tracers allows to obtain semi-quantitative information of a flow field (RAFFEL et al. 2007). For the production of tracer particles, a Rosco 1700 Fog Machine is positioned after the test section, inside the tunnel. The system of image acquisition and image processing to calculate the speed of the flow system consists of an infra-red camera. Tracer droplets are added to the flow by the fog machine, and it is assumed that these particles move uniformly in the flow. As the droplets evaporate over time in these tiny regions local temperature changes, these changes are manifest in the emissivity change in the infra-red and can be recorded with a $60 \mathrm{~Hz}$ frame rate by thermography techniques. The movement of the particles is determined from the analysis of the images using standard correlation methods.

\section{Compatibility of model and real scale}

One remarkable feature of geophysical fields affected by turbulence is that they at least partially show selfsimilar behaviour. This property is manifest in an affine part of the spectrum when represented in double logarithmic form and indicates that there is variability on a range of scales, where the amplitude of the fluctuations at a particular scale is a fixed fraction of the amplitude of fluctuations at a multiple of that scale. This fact together with turbulence production at a largest scale present and the turbulent energy dissipation at the smallest scale present shall be sufficient motivation to justify the kinetic energy spectrum as the key issue to establish relations between wind tunnel experiments and real scale observations, where as one difference among possible others the scales of turbulence production may be cited. In the following we comment on the determination and characteristics of the kinetic energy spectrum.

Evolutions of the micro-meteorological parameters that need the estimation of turbulence statistics are typically 
determined using data windows of the order of an hour in order to make feasible the mean value removal. The windows are centred at a given data point which advances with approximately minute length time steps for each series. For convenience that simplifies the data analysis, the individual wind vectors are rotated into the mean wind direction in such a way that $\mathrm{v}=\mathrm{w}=0$. Then, the mean values of wind speed and temperature, surface layer turbulent statistics $\left(u_{*_{0}}, \overline{\left.w^{\prime} \theta^{\prime}, L\right)}\right.$ and standard deviations $\sigma_{i} \quad(i=u, v, w)$ of the turbulent velocities are calculated. Here $u_{* 0}$ is the surface friction velocity, $\overline{w^{\prime} \theta}$ is the surface sensible turbulent heat flux and $L$ is the Obukhov length scale.

For characterizing the surface layer structure, turbulent fluxes of heat and momentum are estimated using a technique known as eddy covariance. The scaling of velocity (surface friction velocity) is then evaluated from field experiments at a height that corresponds to $10 \mathrm{~m}$ and is defined by

$$
u_{* 0}=\left(\overline{w^{\prime} u^{\prime}}+\overline{w^{\prime} v^{\prime}}\right)^{1 / 4},
$$

where $\overline{w^{\prime} u^{\prime}}$ and $\overline{w^{\prime} v^{\prime}}$ are the surface turbulent momentum fluxes in the direction of the $u$ and $v$ components of the wind vector velocity, respectively. A parameter that is related to the stability regime of surface turbulence is the Obukhov length scale $L$, which is obtained for each run according to

$$
L=\frac{\rho c_{p} u_{* 0}^{3}}{k\left(g / T_{0}\right) \overline{w^{\prime} \theta^{\prime}}},
$$

where $k$ is the von-Karman constant, $g / T_{0}$ is the buoyancy parameter with $g$ the gravitational acceleration and $T_{0}$ the mean surface temperature, $c_{p}$ is the specific heat at constant pressure and $\rho$ is the air density.

The spectra may be computed using well established Fast-Fourier techniques, where details may be found in Stull (1988). The $u$ (longitudinal), $v$ (lateral) and $w$ (vertical) spectra are normalized by $u_{* 0}^{2} \varphi_{\varepsilon}^{2 / 3}$, here $\varphi_{\varepsilon}^{2 / 3}$ is the dimensionless dissipation rate of turbulent kinetic energy and is defined as

$$
\varphi_{\varepsilon}=\frac{k z \varepsilon}{u_{* 0}^{3}}
$$

where $\varepsilon$ is the mean turbulent kinetic energy dissipation per unit time and per unit mass of the fluid, with the order of magnitude of $\varepsilon$ determined only by those quantities that characterize the energy-containing eddies. Following this framework for setting spectral models and the observed turbulent energy spectra in the surface layer (Olesen et al., 1984), when neutral spectra are plotted with a log-log scale against the dimensionless frequency $f=n z / U$, with $n$ being the cyclic frequency, $U$ be mean wind speed and $z$ the observation height, the spectra are characterized by a low-frequency slope of +1 and by a high-frequency slope of $2 / 3$, the latter known as Kolmogorov's law for the inertial subrange (Degrazia et al., 2000)

$$
\frac{n S_{i}}{u_{*}^{2}}=\frac{1.5 c_{i} \varphi_{\varepsilon}^{2 / 3} f}{\left(1+\frac{1.5 f^{5 / 3}}{\left[(f m)_{i}\right]^{5 / 3}}\right)\left[\left(f_{m}\right)_{i}\right]^{5 / 3}}
$$

where $i=u, v, w, u_{*}^{2}=u_{* 0}^{2}(1 z / h)^{1.7}$ is the local friction velocity for a neutral PBL, $\left(f_{m}\right)_{i}$ is the dimensionless frequency of the neutral spectral peak and $c_{i}=\alpha_{i} \alpha_{u}(2 \pi k)^{2 / 3}, \alpha_{u}=0.5 \pm 0.05$, and $\mathrm{m} \alpha_{i}=1,4 / 3,4 / 3$

for the components $u, v, w$ respectively. The peak frequencies $\left(f_{m}\right)_{u},\left(f_{m}\right)_{v}$ and $\left(f_{m}\right)_{w}$ are significant for studies of scalar turbulent transport in the PBL. Especially for the neutral case, these peak frequencies describing the spatial and temporal characteristic of the energy-containing eddies, are expressed by [Tennekes, 1982; Hanna et al.1982]

$$
\left(f_{m}\right)_{i}=\left(f_{m}\right)_{0 i}\left(1+0.03 a_{i} \frac{f_{c} z}{\left(u_{*}\right)_{0}}\right)
$$

where $\left(f_{m}\right)_{0 i}$ is the frequency of the spectral peak at the surface, $f_{c}=10^{-4} s^{-1}$ is the Coriolis parameter. The simplest measure of turbulent velocity fluctuation levels are the variances or mean-square fluctuations $\sigma_{u}^{2}, \sigma_{v}^{2}, \sigma_{w}^{2}$. These parameters represent quantities that allow to estimate the turbulence intensity. Results for these quantities published in Panofsky and Dutton ( 1984), and applied in situations of stability in which mechanical turbulence prevails, suggest the following values: $\quad \sigma_{u}=2.40 u_{*_{0}}, \sigma_{v}=1.90 u_{*_{0}}$ and $\sigma_{w}=1.90 u_{*_{0}}$. Note, that in order to calculate the above velocity variances from the observed turbulent spectra, the knowledge of values of the dimensionless dissipation rate, $\varphi_{\varepsilon}$ is mandatory. Thus, the dissipation rate of turbulent kinetic energy for each run shall be obtained from the inertial sub-range of the one-dimensional spectra of vertical velocity fluctuations using Kolmogorov's law. At neutral stability one expects that $\varphi_{\varepsilon}$ approaches unity due to the balance between shear production and viscous dissipation of turbulence in the absence of any buoyant production and transport.

\section{Conclusion}

As one of the first issues of the STROMA experiment focus is put on the question to establish scaling rules that will determine the relation between findings obtained by simulations in wind tunnel experiments to those of real scale flows. From a theoretical point of view, so far there does not exist any approach that could lead to a genuine theory for turbulence. One of the major challenges in such a direction stems from the fact that scaling has to be established for mean values, variances and all remaining statistical moments for the characteristic distributions that make up turbulence. Hence, according to our reasoning it seems to be safe to start from spectral properties together with the finding 
that for a fully developed turbulence the spectrum shows a universal form. It is noteworthy, that the spectra for the real scale as well as for the model scale (i.e. wind tunnel scale) may be measured with almost the same experimental techniques.

Due to the fact that turbulence is a highly non-linear phenomenon, a universal scale is not likely to exist. However, it is plausible that scales as derived from turbulent spectral data may provide a scale function that for specific spectral ranges relate the turbulent flow of wind tunnel experiments to micro-meteorological wind fields in the planetary boundary layer. In parallel to the present project, another proposal for field experiments will be put into practice, that will among others specify turbulence by its spectral properties from real scale field experiments. Comparisons between the wind tunnel findings and the measurements and observations from micro-meteorology will then shed light on the relation between the two afore discussed scales.

Acknowledgements: The project STROMA is supported by Brazilian Research Agency Conselho Nacional de Desenvolvimento Científico e Tecnológico (CNPq).

\section{References}

Bruun, H. H.,1996. Hot-wire anemometry: principles and signal analysis. Measurement Science and Technology, IOP Publishing.

Degrazia, G. A., Anfossi D., Carvalho J.C., Mangia C., Tirabassi T., Campos Velho H.F., 2000. Turbulence parameterization for $\mathrm{PBL}$ dispersion models in all stability conditions, 34: 3575-3583.

Duda, F. P.; Ramos, F.; Rosa, R.; Temam, R.; Moriconi, L.; Loureiro, J. B. R.; Neto, J. L. da S.; Pinho, F. T.; Cruz, D.; Fontoura, J. L.; Alho, A. T. P.; Ilha, A., 2006. Turbulência: Anais da V Escola de Primavera em Transição e Turbulência. 1. ed. Brasil: Gráfica Graffito, 99-167.

Hanna, S.R.,1982. Applications in air pollution modeling. In: Nieuwstadt, F.T.M., van Dop, H. (Eds.), Atmospheric Turbulence and Air Pollution Modelling. Reidel, Dordrecht, 275-310.

Lienhard, J. H.; Atta, V.,1990. The decay of turbulence in thermally stratified flow. Journal of Fluid Mechanics, Cambridge Univ Press, 210: 57-112.

Ohya, Y.,2001. Wind-tunnel study of atmospheric stable boundary layers over a rough surface. Boundary-Layer Meteorology, Kluwer Academic Publishers,98: 57-82.

Ohya, Y.; Nakamura, R.; Uchida, T., 2008 Intermittent bursting of turbulence in a stable boundary

layer with low-level jet. Boundary-layer meteorology, Springer, 126: 349-363.

Ohya, Y.; Neff, D. E.; Meroney, R. N., 1997 Turbulence structure in a stratified boundary layer under stable conditions. Boundary-Layer Meteorology, Springer, 83:139-162
Olesen, H. R., Larsen, S. E. and Hojstrup, J. 1984. Modelling velocity spectra in the lower part of the planetary boundary layer. Boundary layer Meteorology, 29: 285-312.

Panofsky, H. A. and Dutton, J. A., 1984. Atmospheric Turbulence. Wiley-Interscience, New York.

Raffel, M.; Willert, C.; Wereley, S. T.; Kompenhans, J., 2007. Particle image velocimetry: a practical guide. 2 . ed. Berlin: Springer.

Stull, R. B.1988. An introduction to Boundary Layer Meteorology. 2nd ed Kluwer academic publishers, Dordrecht Netherlands.

Tennekes, H., 1982. Similiraty relations scaling laws and spectral dynamics.In: Nieuwstadt, F.T.M., Van Dop, H. (Eds.), Atmospheric Turbulence and Air Pollution Modelling. Reidel, Dordrecht,37-68.

Yoon, K.; Warhaft, Z.,1990. The evolution of gridgenerated turbulence under conditions of stable thermal stratification. Journal of Fluid Mechanics, Cambridge Univ Press,215: 601-638. 